\title{
Desarrollo y pruebas psicométricas del Instrumento "cuidar" - versión corta para medir la competencia de cuidado en el hogar
}

\author{
Development and psychometric tests of the "cuidar" \\ instrument - short version for the assessment \\ of the competence for home care
}

\author{
Gloria Mabel Carrillo González ${ }^{1}$, Beatriz Sánchez Herrara ${ }^{1}$, Elizabeth Vargas Rosero ${ }^{1}$
}

Forma de citar: Carrillo González GM, Sánchez Herrara B, Vargas Rosero E. Desarrollo y pruebas pscométricas del Instrument "cuidar" - versión corta para medir la competencia del cuidado en el hogar. Rev Univ Ind Santander Salud. 2016; 48(2): 222-231. DOI: http://dx.doi.org/10.18273/revsal.v48n2-2016007 @() (1)

\section{RESUMEN}

Introduccion: Se requiere contar con escalas que midan la competencia para el cuidado en el hogar de pacientes con enfermedades crónicas por cuanto estos reciben excesivos lineamientos para su cuidado y se enfrentan a terapéuticas en extremo complejas. Objetivo: Desarrollar y realizar pruebas psicométricas del instrumento "CUIDAR" versión abreviada para valorar la competencia para el cuidado en el hogar de una persona con enfermedad crónica. Método: Estudio transversal de validación de instrumento en el que a partir de la versión original de la escala "CUIDAR" se desarrolló su versión abreviada, se realizaron pruebas de validez facial y de contenido por medio de panel de expertos, validez de constructo con análisis factorial exploratorio y pruebas de confiabilidad mediante análisis de consistencia interna con el alfa de Cronbach. Resultados: El instrumento "CUIDAR" que mide la competencia de una persona con enfermedad crónica para el cuidado en el hogar en su versión abreviada consta de 20 ítems que incluyen seis dimensiones: el Conocimiento, la Unicidad o condiciones personales para el cuidado, la Instrumental o procedimental, el Disfrute o condiciones mínimas de bienestar, la Anticipación o capacidad de prever e identificar situaciones de alarma y la Relación e Interacción social o red de apoyo con que cuenta el paciente. Su estratificación tiene niveles bajo, medio y alto. La validez con expertos evidenció claridad, coherencia, suficiencia y relevancia de la escala. El análisis factorial ratificó sus seis componentes mediante la rotación Varimax con asociación de los ítems en las mismas categorías propuestas en la versión original. El alfa de Cronbach fue de 0.928. Conclusiones: El Instrumento "CUIDAR” versión abreviada evalúa integralmente la competencia para el Cuidado en el hogar de una persona con enfermedad crónica, demostró validez de contenido, de constructo y alta consistencia interna para su uso en el contexto colombiano.

Palabras clave: Enfermedad crónica, psicometría, cuidados de enfermería en el Hogar, validez de las pruebas, reproducibilidad de resultados

1. Universidad Nacional de Colombia. Bogotá, Colombia

Correspondencia: Gloria Mabel Carrillo González. Dirección: calle 44 45-67 Bloque B2 Of. 801. Correo electronico: gmcarrillog@ unal.edu.co. Teléfono: (+1) 3165000 ext. 10332 


\section{ABSTRACT}

Introduction: It is required to have scales that measure competency for home care of patients with chronic illness because they receive excessive guidelines for their care and they face extremely complex therapeutics. Objective: To develop and carry out psychometric tests of the instrument "CUIDAR" shortened version, to assess competition for home care of a person with chronic illness. Method: Cross-sectional study of validation of the abbreviated version of the scale "CUIDAR" facial validity tests and content through expert panel, construct validity with exploratory factor analysis and reliability testing using analysis of internal consistency with Cronbach's alpha were conducted. Results: The "CUIDAR" instrument that measures the competence of caring for a person with chronic illness at home in its abbreviated version consists of 20 items that include six dimensions: knowledge, Singleness or personal care conditions, Instrumental or procedural aspects of care, Minimum welfare conditions, Anticipation or ability to anticipate and the Social interactions or social support network available for the patients care. The instrument stratification included low, medium and high levels of the care competence. The validity with experts showed clarity, consistency, sufficiency and relevance of the tool. Factor analysis confirmed its six components by Varimax rotation with association of the items in the same categories proposed in the original version. Cronbach's alpha was 0.928. Conclusions: The instrument "CUIDAR" short version fully evaluated competing for home care of a person with chronic disease. It demonstrated content validity as well as construct and high internal consistency for its use in the Colombian context.

Keywords: Chronic disease, psychometrics, home health nursing, validity of tests, reproducibility of results.

\section{INTRODUCCIÓN}

En los últimos años se ha generado una alarma mundial por el incremento de las enfermedades crónicas, en especial en los países en desarrollo ${ }^{1}$. Estas enfermedades son complejas, se prolongan en el tiempo y por lo general demandan apoyos de cuidado $^{2}$; implican la oferta a los pacientes de un cuidado seguro, continuo e integral en ambientes intra y extrainstitucionales ${ }^{3}$. El incremento del costo institucional presiona el paso de servicios de mayor a menor complejidad y de la institución al hogar, sin que exista la preparación adecuada para asumir esta responsabilidad de cuidado ${ }^{4}$. La valoración de la competencia para el cuidado en el hogar de personas con enfermedad crónica y cuidadores familiares dentro de los planes de transición y egreso hospitalario, restringido por algunos al plan de alta hospitalaria, adquiere relevancia en este contexto y en particular en los sistemas de salud.

Al respecto, Hany et al..$^{5}$, señalan que el plan de egreso se ha convertido en un problema central dentro del sistema de salud en donde la estadía hospitalaria tiende a ser más corta, los costos más altos y en consecuencia se incrementa el énfasis en el cuidado comunitario. En tal sentido el plan de egreso es fundamental y debe ser asumido por las enfermeras clínicas. En la medida en que se comprenda la importancia de dicho plan de egreso, se podrán disminuir las readmisiones, los costos del servicio, el estrés para los pacientes y mejorar la continuidad del cuidado.

A partir de una revisión integrativa de literatura que da cuenta del estado actual de conocimiento en la competencia para el cuidado en el hogar, ya publicada por los autores, se identifican estudios que presentan el desarrollo de instrumentos que respaldan la valoración y medición de aspectos a mejorar en el enfermo y sus familiares dentro del plan de egreso, se describen algunas escalas para orientar o medir el plan de transición y el de egreso, enfocadas parcialmente en características de seguridad y continuidad. La revisión no encontró reporte de instrumentos disponibles para este propósito desde una perspectiva integral con pruebas psicométricas en idioma español ${ }^{6}$.

De acuerdo con estos hallazgos, se identificó que la competencia para el cuidado en el hogar es un aspecto fundamental a fortalecer por parte del equipo de salud en las instituciones, siendo su valoración y diagnóstico particularmente necesarios para los pacientes con enfermedades crónicas por cuanto estos reciben excesivos lineamientos para su cuidado y se enfrentan a terapéuticas en extremo complejas. Una vez determinada su relevancia,se procedió a desarrollar teóricamente el constructode la competencia para el cuidado en el hogar, a partir de: 1) Revisión de la literatura, 2) Análisis de resultados de programas para asistir a enfermos crónicos y cuidadores familiares en América Latina y, 3) Exploración de la clasificación de resultados de enfermería (NOC) relacionados con el cuidador familiar y egreso ${ }^{7}$.

La competencia para el cuidado en el hogar se definió teóricamente como la capacidad, habilidad y preparación que tiene la persona con enfermedad crónica $\mathrm{y} / \mathrm{o}$ el cuidador familiar para ejercer la 
ejercer la labor de cuidar en el hogar. Comprende seis categorías que se ordenan bajo el acróstico CUIDAR (por su fácil recordación): Conocimiento, Unicidad (condiciones personales), Instrumental, Disfrutar (bienestar), Anticipación, y Relación social e interacción. Posteriormente, se desarrollaron dos versiones: una para el paciente con enfermedad crónica y otra para el cuidador familiar. Cada versión consta de seis dimensiones con 60 ítems que se miden en escala tipo likert, con puntajes posibles de uno (nunca) a cuatro puntos (siempre o casi siempre), para ser autoadministrados por los participantes?

El uso de estos instrumentos en la práctica clínica, por parte del equipo de salud en 330 personas con enfermedad crónica de todas las cinco macro regiones geográficas colombianas, ha demostrado orientar la planificación de la intervención y la medición del impacto del plan de transición y egreso hospitalario en los pacientes. Sin embargo, su evaluación demostró la necesidad de contar con una versión abreviada que permita optimizar el tiempo requerido para su aplicación y que a la vez mantenga su validez y confiabilidad. En tal sentido, el presente trabajo buscó desarrollar una versión abreviada del instrumento "CUIDAR" a la cual se le realizaron pruebas psicométricas para valorar la competencia para el cuidado en el hogar de una persona con enfermedad crónica.

\section{METODOLOGÍA}

Estudio transversal de validación de instrumentos, desarrollado entre los años 2012 - 2014, y que contó con el aval del Comité de ética de la Facultad de Enfermería de la Universidad Nacional de Colombia en el marco del Programa para disminuir la carga de la Enfermedad Crónica en Colombia, mantuvo todos los requisitos exigidos por la Norma 8430 de 1993, por la cual se establecen las pautas científicas, técnicas y administrativas para la investigación en salud en Colombia y acogió la política de protección ambiental ${ }^{8}$. Comprendió tres fases:

1. Desarrollo de la versión abreviada. Con la asesoría de tres expertos, en estadística y psicometría, se determinó como primer criterio para la reducción de ítems la información que proporciona la matriz anti-imagen y los pesos factoriales de cada ítem, con base en la información obtenida en la aplicación de la versión original a personas con enfermedad crónica. Posteriormente, los autores revisaron los ítems a conformar la versión abreviada, contrastando el sentido y constructo de cada dimensión con cada uno de los ítems seleccionados buscando que estos dieran la mayor representación de la categoría. Así mismo, se revisó la pertinencia de la medición a través de escala tipo Likert con puntajes de 1 a 4 y se ajustó de 0 a 3 ( 0 nunca y 3 siempre o casi siempre).

2. Validez facial y de contenido: Con el fin de determinar si la versión abreviada del instrumento realmente medía lo que se proponía medir, se recurrió a la validez facial y de contenido con un panel de expertos, cuyos criterios de inclusión fueron tener posgrado en salud a nivel de especialización, maestría o doctorado y contar con 10 o más años de experiencia trabajando en el campo del cuidado a personas con enfermedad crónica o áreas afines. Los expertos revisaron los ítems en cuanto a coherencia, suficiencia, claridad, relevancia e importancia de acuerdo a lo propuesto por Lawshe modificado por Tristan9. Para determinar el grado de acuerdo entre jueces se utilizó el índice de concordancia Kappa de Fleiss ${ }^{10}$, considerando la fuerza de concordancia como aceptable cuando los índices kappa se encontraran entre 0.41 y 0.60 y buena o muy buena si estos daban valores mayores a 0.61 .

La obtención del índice de contenido se basó en el modelo de Lawshe modificado 9 , que representa una propuesta de comprobación útil para la valoración de la validez de contenido de las pruebas diseñadas, adaptadas o utilizadas en salud. En cada ítem se calculó la razón de validez de contenido modificada (CVR) y para todo el instrumento se obtuvo el índice de validez de contenido (CVI).

1. Validez de constructo y confiabilidad: Para determinar la validez de constructo y la confiabilidad se aplicó la versión abreviada del instrumento a personas con enfermedad crónica. El cálculo de tamaño de la muestra requerido se obtuvo de acuerdo al número de ítems del instrumento, considerando un mínimo de 600 participantes (20 por ítem) $)^{11,12}$

Los criterios de inclusión de los participantes fueron ser personas con enfermedad crónica diagnosticada, mayores de 18 años, que supieran leer y escribir y que asistieran a una institución prestadora de servicios de salud.

La validez de constructo, se realizó por medio de la técnica de análisis factorial exploratorio utilizando el método de componentes principales y rotación varimax. 
Se acogió lo descrito por Nunally y Bernstein ${ }^{13}$ respecto a los criterios en la evaluación de los ítems del instrumento con presencia de una carga factorial (loading) igual o superior a 0.3 con comunalidad al menos de 0.1. y en primera instancia ausencia de efecto adverso sobre las medidas de confiabilidad calculadas mediante el índice de Cronbach-Mesbah ${ }^{14}$, y en segunda, ausencia de correlación fuerte (superior a 0.8) y significancia con los demás ítems.

Para determinar la confiabilidad por medio de su nivel de consistencia interna se empleó el alfa de Cronbach ${ }^{15}$ A continuación se ilustra la metodología del estudio de manera esquemática. (Figura 1).

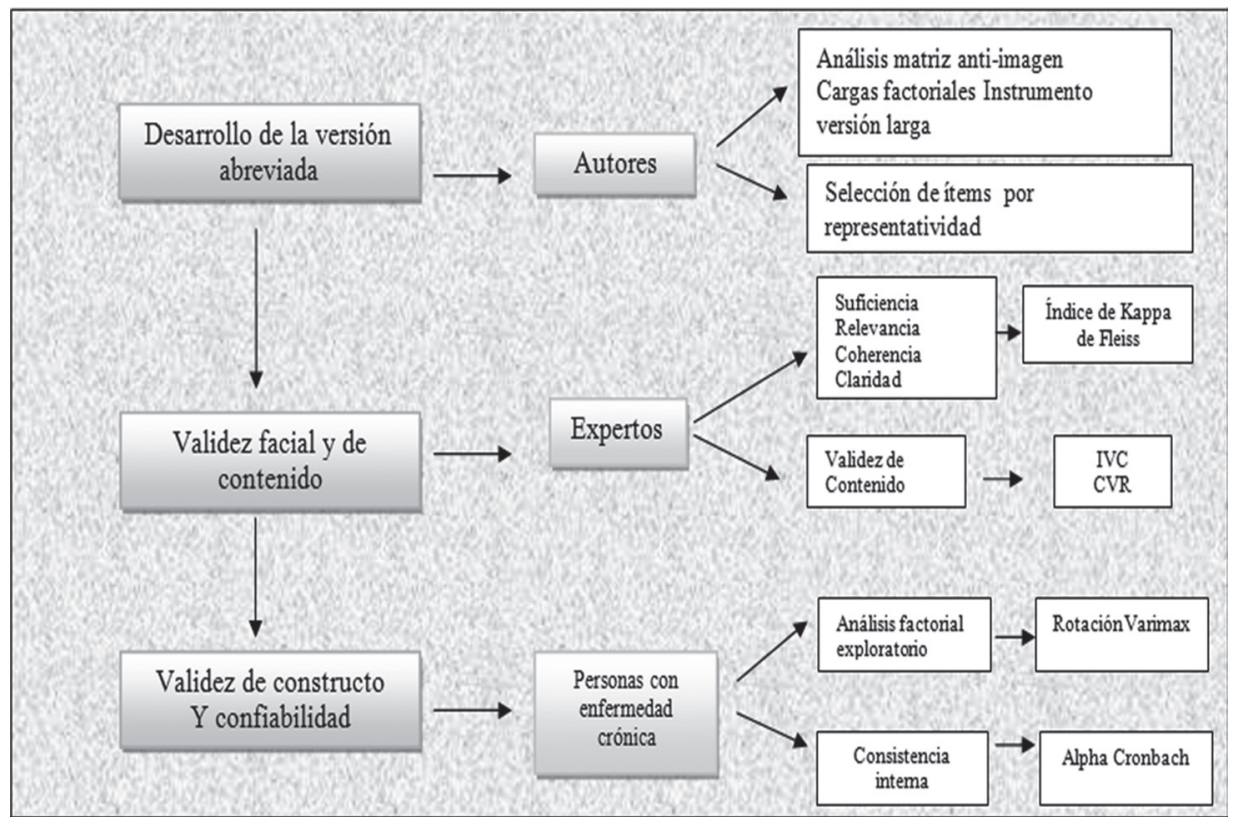

Figura 1. Esquema de la Metodología

Fuente: Elaboración de las autoras. 2015

\section{RESULTADOS}

1. Desarrollo del instrumento. La versión abreviada del instrumento "CUIDAR"se conformó a partir de los criterios descritos en la metodología. Consta de 20 ítems que se miden a través de escala tipo Likert con puntuaciones que van de 0 a 3; 0 es nunca, 1 pocas veces, 2 con frecuencia y 3 casi siempre o siempre. La estratificación de los rangos alto, medio y bajo fue resultado del análisis estadístico y la aplicación de la regla de Dalheinius.

2. Validez de contenido. Ocho expertos participaron en el proceso de validez facial y de contenido. Se obtuvo una fuerza de concordancia entre aceptable y muy buena por parte de los jueces; aceptable en cuanto a claridad $(0,57)$, y muy buena en lo concerniente a coherencia $(0,66)$, relevancia $(0,66)$ y suficiencia $(0,61)$. Hallazgos que permiten afirmar que el instrumento en su versión abreviada es suficiente, claro, coherente y relevante en la medición de la competencia para el cuidado en el hogar de personas con enfermedad crónica.
Índice de Contenido: El CVI del instrumento en su medición global fue de 0.97 , para los ítems se obtuvieron índices entre 0.88 y 1.00 , hallazgo que indica que la validez de contenido del instrumento es muy buena.

3. Validez de constructo y confiabilidad. El instrumento en su versión abreviada se aplicó a 959 personas con Enfermedades Crónicas No Transmisibles (ECNT) de las cinco macro regiones geográficas del país. La mayoría de los enfermos crónicos participantes del estudio son del género femenino, de todas las edades predominando los que se encuentran entre los 36 a 74 años, con grados de escolaridad bajos especialmente, de procedencia urbana y ocupación hogar. Dentro de la morbilidad reportada se encuentra en mayor proporción las enfermedades cardiovasculares, seguidas por el cáncer, la diabetes, la enfermedad renal y las de tipo osteoarticular. Gran porcentaje de los participantes tiene estado mental intacto, bajos niveles de dependencia y más de 36 meses con el diagnóstico de la entidad patológica. (Tabla 3) Caracterización sociodemográfica de los participantes 
Tabla 1. Instrumento "CUIDAR" - Versión abreviada por dimensiones y rangos)

\begin{tabular}{|c|c|c|c|c|}
\hline & DESCRIPCIÓN & $\begin{array}{c}\text { NO. ÍTEMS/ } \\
\text { PUNTAJES POSIBLES }\end{array}$ & \multicolumn{2}{|c|}{ CLASIFICACIÓN } \\
\hline $\begin{array}{l}\text { Dimensión } \\
\text { Conocimiento }\end{array}$ & $\begin{array}{l}\text { Nociones e ideas respecto a la enfermedad, } \\
\text { las terapéuticas farmacológicas y no } \\
\text { farmacológicas, las indicaciones especiales } \\
\text { de la terapéutica, el manejo de dispositivos y } \\
\text { equipos durante el egreso; conocimiento de las } \\
\text { actividades a realizar propias de la cotidianidad } \\
\text { tales como baño, vestido, alimentación, } \\
\text { deambulación, entre otras }\end{array}$ & $\begin{array}{c}3 \\
(0-9)\end{array}$ & Medio & $4-6$ \\
\hline $\begin{array}{l}\text { Dimensión } \\
\text { Unicidad }\end{array}$ & $\begin{array}{l}\text { Características tanto internas como de } \\
\text { personalidad, base fundamental para que se } \\
\text { afronte de una u otra forma la enfermedad } \\
\text { crónica y el rol como persona enferma o } \\
\text { cuidador. Herramientas con las que se cuenta } \\
\text { para hacer frente a problemas, retos y desafíos, } \\
\text { así como mecanismos de afrontamiento para } \\
\text { superarlos de la mejor manera. }\end{array}$ & $\begin{array}{c}4 \\
(0-12)\end{array}$ & Medio & $10-12$ \\
\hline $\begin{array}{l}\text { Dimensión } \\
\text { Instrumental }\end{array}$ & $\begin{array}{l}\text { Habilidades y destrezas para realizar las labores } \\
\text { de cuidado, que incluyen la administración de } \\
\text { medicamentos, satisfacción de necesidades } \\
\text { básicas de la vida diaria como alimentación, } \\
\text { eliminación, deambulación, baño y vestido, } \\
\text { entre otras. }\end{array}$ & $\begin{array}{c}3 \\
(0-9)\end{array}$ & $\begin{array}{l}\text { Medio } \\
\text { Alto }\end{array}$ & $\begin{array}{l}6-7 \\
8-9\end{array}$ \\
\hline $\begin{array}{l}\text { Dimensión } \\
\text { Disfrutar }\end{array}$ & $\begin{array}{l}\text { Grado de bienestar que se tiene para comprender } \\
\text { cómo se asume la responsabilidad del cuidado } \\
\text { en casa. Se indagan aspectos básicos de la vida } \\
\text { cotidiana, así como la satisfacción personal } \\
\text { relacionada con la calidad de vida percibida. }\end{array}$ & $\begin{array}{c}4 \\
(0-12)\end{array}$ & Medio & $\begin{array}{l}6-8 \\
9-12\end{array}$ \\
\hline $\begin{array}{l}\text { Dimensión } \\
\text { Anticipación }\end{array}$ & $\begin{array}{l}\text { Nociones e ideas que se tienen, pensando en } \\
\text { anticiparse a todas aquellas necesidades que } \\
\text { pueda presentar la persona con enfermedad } \\
\text { crónica en un futuro, la planeación ante } \\
\text { la ocurrencia de un evento inesperado, la } \\
\text { preparación para un examen y la identificación } \\
\text { de riesgos, entre otros. }\end{array}$ & $\begin{array}{c}2 \\
(0-6)\end{array}$ & Medio & $\begin{array}{l}3-4 \\
5-6\end{array}$ \\
\hline $\begin{array}{l}\text { Dimensión } \\
\text { Relación e } \\
\text { Interacción }\end{array}$ & $\begin{array}{l}\text { Relaciones sociales y las redes de apoyo } \\
\text { primarias y secundarias que se tienen. Se } \\
\text { constituyen como factores que favorecen el } \\
\text { fortalecimiento de la labor de cuidado }\end{array}$ & $\begin{array}{c}4 \\
(0-12)\end{array}$ & Medio & $\begin{array}{l}8-10 \\
11-12\end{array}$ \\
\hline $\begin{array}{l}\text { Competencia para } \\
\text { el cuidado en el } \\
\text { Hogar (CUIDAR) }\end{array}$ & $\begin{array}{l}\text { Capacidad, habilidad y preparación que } \\
\text { tiene la persona con enfermedad crónica y/o } \\
\text { el cuidador familiar para ejercer la labor de } \\
\text { cuidar en el hogar. }\end{array}$ & $\begin{array}{c}20 \\
(0-60)\end{array}$ & Medio & $49-60$ \\
\hline
\end{tabular}

Fuente: Datos del estudio, 2015 
Salud Vol.48 No.2 Abril - Junio de 2016

Tabla 2. Caracterización sociodemográfica de los participantes

\begin{tabular}{|c|c|c|}
\hline \multicolumn{2}{|c|}{ CARACTERÍSTICA } & $\begin{array}{c}\text { Porcentaje \% } \\
\quad(n=959)\end{array}$ \\
\hline \multirow{2}{*}{ GÉNERO } & Masculino & 40,26 \\
\hline & Femenino & 59,74 \\
\hline \multirow{4}{*}{ EDAD } & 18 a 35 & 20,60 \\
\hline & 36 a 59 & 32,60 \\
\hline & 60 a 74 & 32,40 \\
\hline & Mayor de 75 & 14,40 \\
\hline \multirow{3}{*}{ ESCOLARIDAD } & Baja & 58,60 \\
\hline & Media & 26,40 \\
\hline & Alta & 15,00 \\
\hline \multirow{2}{*}{$\begin{array}{c}\text { ZONA DE } \\
\text { PROCEDENCIA }\end{array}$} & Rural & 16,80 \\
\hline & Urbana & 83,11 \\
\hline \multirow{5}{*}{ ESTADO CIVIL } & Casado(a) & 30,90 \\
\hline & Separado(a) & 7,60 \\
\hline & Soltero(a) & 29,00 \\
\hline & Unión Libre & 15,40 \\
\hline & Viudo(a) & 17,10 \\
\hline \multirow{6}{*}{ OCUPACIÓN } & Empleado(a) & 11,60 \\
\hline & Estudiante & 0,90 \\
\hline & Hogar & 57,90 \\
\hline & Independiente & 20,40 \\
\hline & Ninguna & 6,40 \\
\hline & Pensionado & 2,90 \\
\hline \multirow{3}{*}{$\begin{array}{c}\text { NIVEL DE } \\
\text { FUNCIONALI-DAD } \\
\text { SEGUN MEDICIÓN } \\
\text { CON ESCALA PULSES }\end{array}$} & Dependencia Baja & 67 \\
\hline & $\begin{array}{l}\text { Dependencia } \\
\text { moderada }\end{array}$ & 23 \\
\hline & Dependencia total & 11 \\
\hline \multirow{13}{*}{$\begin{array}{l}\text { MORBILIDAD } \\
\text { REPORTADA }\end{array}$} & Otras & 17,80 \\
\hline & Autoinmune & 0,50 \\
\hline & Cardiovascular & 29,1 \\
\hline & Sistema circulatorio & 0,50 \\
\hline & Hepática y/o biliar & 0,50 \\
\hline & Mental & 1,30 \\
\hline & Diabetes & 13,4 \\
\hline & Neurológica & 5,00 \\
\hline & Cáncer & 15,1 \\
\hline & Osteoarticular & 9,10 \\
\hline & Renal & 4,10 \\
\hline & Respiratoria crónica & 1,70 \\
\hline & $\begin{array}{c}\text { Secuelas de } \\
\text { traumatismos }\end{array}$ & 1,80 \\
\hline \multirow{4}{*}{$\begin{array}{l}\text { TIEMPO CON LA } \\
\text { ENFERMEDAD }\end{array}$} & 0 a 6 meses & 20,5 \\
\hline & 7 a 18 meses & 12,1 \\
\hline & 19 a 36 meses & 18,0 \\
\hline & más de 36 meses & 49,4 \\
\hline
\end{tabular}

Fuente: Datos del estudio, 2015
Con respecto a la Validez de constructo y en concordancia con el método descrito se identificó el cumplimiento de tres condiciones necesarias para realizar el análisis factorial: Se obtuvo una muestra de personas con enfermedad crónica mayor de 100 ( $\mathrm{n}=958$ participantes); un índice de medida de adecuación de la muestra (KMO), $\geq 0.9$, lo que indica que análisis factorial es pertinente para la muestra obtenida; y prueba de esfericidad de Barlett significativa valor de $\mathrm{p}<0.00$ indicando existencia de correlación entre los factores lo que permite el análisis factorial.

El análisis del efecto de los ítems sobre la validez de constructo del instrumento mostró que todos los ítems presentaban pesos factoriales y comunalidad con valores muy superiores al límite inferior permitido (se consideraron significativos los pesos factoriales superiores a 0,3 y/o los valores de comunalidad mayor a 0,1$)$. Se resalta, además, que tres ítems $(7,9$ y 14) reportaron complejidad factorial al presentar valores de comunalidad superiores 0,3 en más de un factor, por lo que se consideró asociarlos con el factor en el que se obtuvo mayor valor.

Teniendo en cuenta que teóricamente el instrumento tiene seis dimensiones, y dejando que el análisis factorial proponga el número de componentes mediante la rotación varimax en las que se asocian la mayor parte de los ítems de acuerdo a las dimensiones propuestas, se obtuvieron seis componentes que incluyen: Conocimiento: los tres ítems con cargas factoriales entre 0.76 y 0.82 ; Unicidad: los cuatro ítems con cargas factoriales entre 0.53 y 0.74 ; instrumental dos de los tres ítems con cargas factoriales entre 0.71 y 0.80 ; Disfrutar: los cuatro ítems se asocian a esta dimensión con cargas factoriales entre 0.65 y 0.81 ; Anticipación: los dos ítems propuestos con cargas factoriales entre 0.75 y 0.76 ; y Relación e interacción social: cuatro ítems se asocian con cargas factoriales entre 0.74 y 0.82 . El método de extracción y el análisis de componentes principales con los seis factores propuestos dieron una varianza total explicada del $72,94 \%$.

La confiabilidad por consistencia interna medida con el alfa de Cronbach fue de 0,928 para el instrumento en su totalidad, con valores entre 0,926 y 0,930 en cada una de las dimensiones: conocimiento 0.929 ; unicidad 0,930 ; instrumental y procedimental 0,926 ; disfrutar 0,928 ; anticipación 0,927 y relación e interacción social 0,927 . 
Desarrollo y pruebas psicométricas del Instrumento "cuidar" - versión corta para medir la competencia de cuidado en el hogar

Tabla 3. Rotación varimax y comunalidad por ítem del instrumento "CUIDAR" - Versión abreviada)

\begin{tabular}{|c|c|c|c|c|c|c|c|c|}
\hline & \multirow{2}{*}{ ITEM } & \multirow{2}{*}{ Comunalidad } & \multicolumn{6}{|c|}{ Componente } \\
\hline & & & 1 & 2 & 3 & 4 & 5 & 6 \\
\hline $\mathrm{CCP} 1$ & Sé cómo hacer seguimiento de mis condiciones de salud & 0,729 & 0,146 & 0,154 & 0,762 & 0,186 & 0,186 & 0,182 \\
\hline $\mathrm{CCP} 2$ & Conozco cómo realizar los procedimientos requeridos para cuidarme & 0,83 & 0,153 & 0,135 & 0,854 & 0,149 & 0,144 & 0,127 \\
\hline $\mathrm{CCP} 3$ & Conozco los signos de alarma de mi condición de salud & 0,767 & 0,104 & 0,182 & 0,829 & 0,162 & 0,088 & 0,039 \\
\hline $\mathrm{CCP} 4$ & Tengo confianza en mi capacidad para asumir mi cuidado en casa & 0,698 & 0,238 & 0,275 & 0,24 & 0,662 & 0,232 & 0,128 \\
\hline CCP5 & Supero fácilmente sentimientos de culpa o rabia & 0,627 & 0,287 & 0,239 & 0,173 & 0,661 & 0,05 & 0,135 \\
\hline CCP6 & Soy capaz de simplificar las tareas del cuidado & 0,754 & 0,206 & 0,236 & 0,206 & 0,746 & 0,164 & 0,173 \\
\hline $\mathrm{CCP} 7$ & Establezco mi propio plan de vida & 0,617 & 0,252 & 0,303 & 0,109 & 0,536 & 0,359 & 0,184 \\
\hline ССР8 & $\begin{array}{l}\text { Actúo siguiendo las recomendaciones prescritas por el equipo de salud } \\
\text { (actividad, dieta, otros) }\end{array}$ & 0,715 & 0,239 & 0,083 & 0,174 & 0,172 & 0,278 & 0,717 \\
\hline ССР9 & $\begin{array}{l}\text { Organizo el apoyo instrumental disponible para mi cuidado (transporte, } \\
\text { equipos, insumos, otros) }\end{array}$ & 0,664 & 0,067 & 0,173 & 0,193 & 0,361 & 0,493 & 0,468 \\
\hline CCP10 & Uso adecuadamente los medicamentos formulados & 0,805 & 0,261 & 0,175 & 0,112 & 0,16 & 0,132 & 0,807 \\
\hline CCP11 & Estoy satisfecho con mi condición de salud actual & 0,762 & 0,091 & 0,815 & 0,213 & 0,145 & 0,051 & 0,141 \\
\hline CCP12 & Siento tener energia para responder a las necesidades de cuidado & 0,707 & 0,18 & 0,689 & 0,187 & 0,298 & 0,125 & 0,248 \\
\hline CCP13 & Siento que mi situación de salud me permite desarrollar mi plan de vida & 0,761 & 0,191 & 0,787 & 0,079 & 0,229 & 0,202 & 0,079 \\
\hline CCP14 & $\begin{array}{l}\text { El cuidado de mi salud no me impide desarrollar otros asuntos a actividades } \\
\text { personales }\end{array}$ & 0,632 & 0,162 & 0,659 & 0,132 & 0,2 & 0,335 & $-0,029$ \\
\hline CCP15 & Preveo manejo de riesgos y de necesidades para mi cuidado & 0,809 & 0,209 & 0,265 & 0,228 & 0,162 & 0,761 & 0,195 \\
\hline CCP16 & Organizo los recursos necesarios para mi cuidado & 0,817 & 0,235 & 0,242 & 0,18 & 0,205 & 0,75 & 0,257 \\
\hline CCP17 & Logro respaldo de otras personas para apoyar mi cuidado & 0,671 & 0,758 & 0,161 & 0,143 & 0,125 & 0,138 & 0,127 \\
\hline CCP18 & Mantengo tranquilidad emocional con las personas que apoyan mi cuidado & 0,662 & 0,741 & 0,191 & 0,078 & 0,119 & 0,123 & 0,201 \\
\hline CCP19 & $\begin{array}{l}\text { Busco comunicarme efectivamente con las personas quienes apoyan mi } \\
\text { cuidado }\end{array}$ & 0,782 & 0,823 & 0,098 & 0,117 & 0,244 & 0,107 & 0,104 \\
\hline CCP20 & Fortalezco vínculos de afecto con las personas que apoyan mi cuidado & 0,78 & 0,819 & 0,102 & 0,123 & 0,226 & 0,121 & 0,133 \\
\hline
\end{tabular}

Tabla 4. Varianza total explicada primeros 10 componentes obtenidos

\begin{tabular}{cccccc}
\hline \multirow{2}{*}{ Componente } & \multicolumn{3}{c}{ Autovalores iniciales } & \multicolumn{2}{c}{$\begin{array}{c}\text { Sumas de las saturaciones al } \\
\text { cuadrado de la extracción }\end{array}$} \\
\cline { 2 - 6 } & Total & \% de la varianza & \% acumulado & Total & \% de la varianza \\
\hline 1 & 8,842 & 44,212 & 44,212 & 8,842 & 44,212 \\
2 & 1,706 & 8,528 & 52,740 & 1,706 & 8,528 \\
3 & 1,392 & 6,960 & 59,700 & 1,392 & 6,960 \\
4 & 1,175 & 5,874 & 65,575 & 1,175 & 5,874 \\
5 &, 798 & 3,988 & 69,563 & & \\
6 &, 672 & 3,362 & 72,925 & & \\
7 &, 571 & 2,855 & 75,780 & & \\
8 &, 530 & 2,648 & 78,429 & & \\
9 &, 524 & 2,618 & 81,046 & & \\
10 &, 480 & 2,400 & 83,446 & & \\
\hline
\end{tabular}

Fuente: Datos del estudio, 2015 


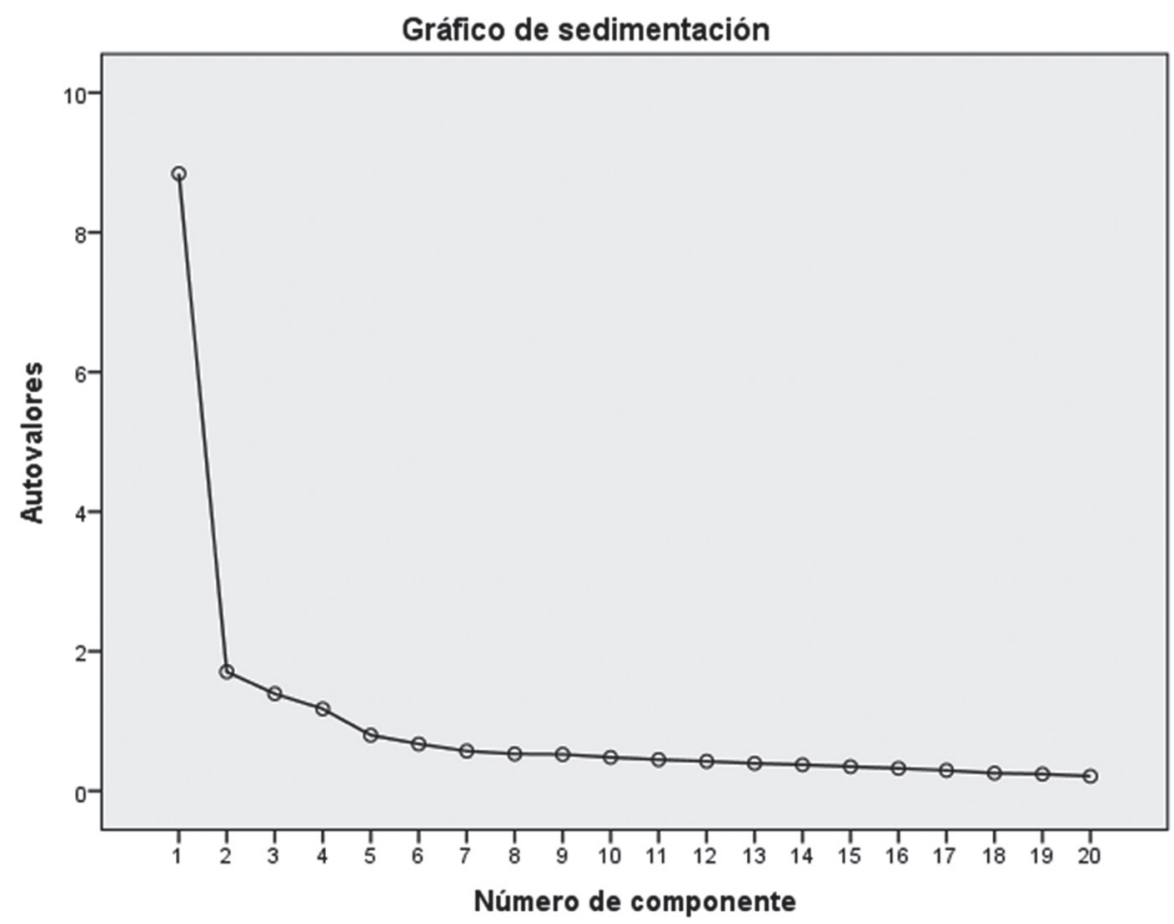

Figura 2. Pendiente de cattell

\section{DISCUSIÓN}

La competencia para el cuidado en el hogar hace parte de los aspectos indispensables en la valoración de personas con enfermedad crónica, con el fin de determinar las necesidades sobre las que se deben orientar los planes de transición y el seguimiento extra hospitalario. Sin embargo, precisar los aspectos y/o dimensiones que debe contener dicha valoración se ha considerado una tarea difícil sin que existan directrices claras al respecto y se evidencia una transición con apoyo insuficiente ${ }^{16}$.

Son varios los estudios que reconocen que el cuidado en casa es cada día más complejo, las instrucciones de salida, su propósito, la definición de las metas de estas instrucciones y planes, los beneficios de escribirlas, la consideración de los obstáculos para comprender la instrucción, la necesidad de establecer con quién debe ser revisada y la pertinencia de los impresos de las instrucciones de salida, deben ser abordadas para generar un plan adecuado. Sin embargo, es determinante perfilar la valoración y definir las metodologías educativas del usuario $^{17-22}$.

El instrumento "CUIDAR" que valora la competencia para el cuidado en el hogar de la persona con enfermedad crónica, aporta conceptualmente en la comprensión de los aspectos básicos a evaluar y se constituye en una herramienta para orientar de forma precisa al equipo de salud. La versión abreviada permite además responder a las necesidades de la clínica en donde el manejo de los tiempos y la carga del trabajo con las personas que tienen enfermedad crónica deben ser considerados como aspectos fundamentales para garantizar la calidad de la atención ${ }^{23,24}$.

La competencia involucra componentes propios del conocimiento de la entidad patológica, los aspectos instrumentales de la terapéutica y la capacidad de anticipación que se tenga para consultar oportunamente minimizando posibles complicaciones y riesgos; sin embargo, se requiere además, enfatizar en cuestiones propias de la personalidad, base fundamental para que se afronte de una u otra forma la enfermedad crónica; en el grado de satisfacción que se tiene para comprender cómo se asume la responsabilidad del cuidado en casa y en la identificación de redes de apoyo primarias y secundarias con las que se cuente. En este sentido, se observa alta concordancia respecto a suficiencia, relevancia y coherencia dada por los expertos al instrumento globalmente respecto a sus dimensiones y los ítems que las conforman.

Al revisar las escalas utilizadas en estudios sobre impacto o efecto de los planes de transición y egreso hospitalario, se identifican diferentes perspectivas que suponen la alta variabilidad sobre las percepciones que tiene el equipo de salud en relación al alcance y el 
foco de interés que representa un plan de egreso. Se puntualiza en aspectos tales como la calidad de vida relacionada con el estado de salud ${ }^{25}$, la percepción del paciente sobre la calidad del cuidado y servicios ${ }^{26}$, la calidad de educación y la preparación para el alta hospitalaria particularmente en el componente instrumental ${ }^{27}$, la valoración de las necesidades de los pacientes de cuidado agudo ${ }^{28}$. En contraste, en la presente investigación los expertos consideraron esenciales y relevantes los ítems y las dimensiones propuestas en el instrumento de competencia para el cuidado en el hogar versión abreviada, aspectos que se reportan por medio del IVC y el CVR obtenidos.

Respecto a la validez de constructo, es posible afirmar que se cuenta con una escala multidimensional y específica que cumple con requerimientos psicométricos para ser utilizada en el contexto colombiano, siendo un referente para la comprensión y conocimiento de la competencia para el cuidado en el hogar de personas con enfermedad crónica, correspondiendo a un aporte clínico con el fin de consolidar estrategias efectivas que la fortalezcan.

Parte de este aporte se da al identificar que el número de factores resultante de la solución del análisis factorial pudo aproximarse al número de dimensiones planteadas en la versión original (larga), las cargas factoriales de los ítems corresponden exactamente con los diseñados y planteados por los autores para medir cada dimensión. Se identifica además la fortaleza de la escala para abordar personas que cursan con diferentes enfermedades crónicas en Colombia, teniendo en cuenta el número de participantes, la captación de usuarios de diferentes regiones del país y con diversas patologías crónicas. Es de anotar el valor obtenido en la confiabilidad, mediante la determinación de un alfa de Cronbach alto, similar a lo reportado por los autores ${ }^{6}$ en la versión original-larga, aspecto que ratifica la consistencia interna de la escala.

La validación de un instrumento es un proceso continuo y dinámico que va adquiriendo más consistencia en cuanto más propiedades psicométricas se hayan medido con distintas poblaciones y sujetos ${ }^{29}$, razón por la cual se considera necesario, para futuros estudios, determinar la estabilidad y sensibilidad de esta versión abreviada en usuarios con enfermedades crónicas a quienes se les genera un plan de egreso orientado a fortalecer cada una de las dimensiones propuestas en la competencia para el cuidado en el hogar.

Teniendo en cuenta que un posible sesgo de selección pudo haber sido las características de la población explorada en relación a la enfermedad crónica, grado de dependencia de los usuarios y escenarios de captación, se espera profundizar en el comportamiento de la escala en general y de los ítems que presentaron complejidad factorial con submuestras de pacientes que tengan diferentes condiciones que pueden afectar su competencia para el cuidado en el hogar, tales como: fase de la enfermedad crónica, comorbilidades, redes de apoyo familiar y social con las que cuenten, y escenario de captación (hospitalizados o consulta externa).

\section{CONCLUSIONES}

El Instrumento "CUIDAR" versión abreviada evalúa integralmente la competencia para el cuidado en el hogar de una persona con enfermedad crónica y demostró validez de constructo y de contenido y alta confiabilidad determinada mediante consistencia interna para ser usado en el contexto colombiano. Esta versión de 20 ítems, reduce la versión original a la tercera parte y mantiene los elementos de suficiencia, coherencia, claridad, relevancia y representatividad del constructo propuesto por los autores en la versión original. El instrumento "CUIDAR" - versión abreviada es una herramienta que responde a las demandas de la clínica en términos de rigor científico y tiempo requerido para la evaluación integral de la competencia del cuidado en el hogar que permite además de conocer su nivel, planificar el cuidado de personas con enfermedad crónica.

\section{REFERENCIAS}

1. Enfermedades Crónicas, Hechos y cifras.

2. Sánchez B. Cuidado seguro: la nueva tendencia en el cuidado de la salud. Rev Actual Enfermer. 2011; 14(2): 27-32.

3. Pinto N, Barrera L, Sánchez B, Figueroa P, Camargo L. Habilidad de cuidado de los cuidadores familiares de personas con enfermedad crónica. Mirada internacional. Aquichan. 2006; 6(1): 22-33.

4. Lin F, Chaboyer W, Wallis M. A literature review of organizational, individual and teamwork factors contributing to the ICU discharge process. Aust Crit Care. 2009; 22(1): 29-43. DOI: 10.1016/j. aucc.2008.11.001.

5. Chin-Yen H, Chin-Yi C, Hui-Ling L. Another compulsory clinical competence for emergency nurses -Implementing effective discharge planning. Austral Emerg Nurs J. 2009; 12(4): 178-186. DOI: 10.1016/j.aenj.2009.08.081.

6. Sánchez B, Carrillo GM, Barrera L. El plan de transición y egreso hospitalario y su efecto en el cuidado de la salud: una revisión integrada. Rev Udca actual Div Cient. 2014; 17(1): 13-23. 
7. Carrillo G, Sánchez B, Arias M. Validation of an instrument to assess the homecare competency of the family caregiver of a person with chronic disease. Invest Educ Enferm. 2015; 33(3): 449-455.

8. Universidad Nacional de Colombia, Consejo Superior Universitario. Acuerdo 016 de 2011 por el cual se establece la Política Ambiental de la Universidad Nacional de Colombia.

9. Tristán A. Modificación de los modelos de Lawshe para el dictamen cuantitativo de la validez de contenido de un instrumento objetivo. Avances en Medición. 2006; 6(1): 37-48.

10. Fleiss J. Measuring nominal scale agreement among many raters. Psycological Bull. 1971; 76(5): 378-382.

11. Argibay JC. Muestra en Investigación Cuantitativa. Subj Procesos Cogn. 2009; 13(1): 13-29.

12. Lévy J, Varela J. Modelización de covarianzas en ciencias sociales. España: Netbiblo. 2006.

13. Nunnally J, Bernestein H. Teoría Psicométrica. Tercera Edición. México: Mc GrawHill. 1995.

14. Mesbah M. Statistical quality of life. Method an Aplications of Statistics in the Life and Health Sciences. Balakrishnan (Editor). New Jersey: Wiley. 2010.

15. Campo-Arias A, Oviedo HC. Propiedades psicométricas de una escala: la consistencia interna. Rev Salud Publica. 2008; 10(5): 831-839.

16. Chen J, Ross JS, Carlson MD, Lin Z, Normand SL, Bernheim SM, et al. Skilled nursing facility referral and hospital readmission rates after heart failure or myocardial infarction. Ame J Med. 2012; 125(1): 100-109. DOI: 10.1016/j.amjmed.2011.06.011.

17. Krohn D. Discharge instructions in the outpatient setting: Nursing considerations. J Radiol Nursing. 2008. 27(1): 29-33. DOI: $10.1016 / j$. jradnu.2007.10.001.

18. Han CY, Barnard A, Chapman H. Discharge planning in the emergency department: a comprehensive approach. J Emerg Nurs. 2009; 35 (6): 525-528. DOI: $10.1016 /$ j.jen.2009.01.015.

19. Collier EJ. Harrington C. Discharge planning, nursing home placement and the Internet. Nurs Outlook. 2005; 53(2): 95-103.

20. Pichitpornchaia W, Street A, Boontong A. Discharge planning and transitional care: issues in Thai nursing. Int J Nurs Stud.1999; 36(5): 355-362.

21. Watts R, Pierson J, Gardner H. How do critical care nurses define the discharge planning process?. Intensive Crit Care Nurs. 2005; 21(1): 39-46.

22. Hanratty B, Holmes L, Lowson E, Grande G, Addington-hall J, Payne S, et al. Older Adults' experiences of transitions between care settings at the end of life in England: a qualitative interview study. J Pain Symptom Manage. 2012; 44(1): 74-83. DOI: 10.1016/j.jpainsymman.2011.08.006.

23. Atefi N, Abdullah KL, Wong LP, Mazlom R. Factors influencing registered nurses perception of their overall job satisfaction: a qualitative study. Int Nurs Rev. 2014; 61(3): 352-360. DOI: 10.1111/inr. 12112.

24. Grau A, Flichtentrei D, Suñer R, Prats M, Braga F. Influencia de factores personales, profesionales y transnacionales en el síndrome de Burnout en personal sanitario hispanoamericano y español. Rev Esp Salud Pública. 2009; 83 (2): 215-230.

25. Annells M, Koch T, Brown M. Client relevant care and quality of life: the trial of a client generated index (CGI) tool for community nursing. Int J Nurs Stud. 2001; 38(1): 9-16.

26. Senarath U, Gunawardena NS. Development of an instrument to measure patient perception of the quality of nursing care and related hospital services at the National Hospital of Sri Lanka. Asian Nurs Res. 2011; 5(2):71-80. DOI: 10.1016/S19761317(11)60015-7.

27. Bobay KL, Jerofke TA, Weiss ME, Yakusheva O. Age-related differences in perception of quality of discharge teaching and readiness for hospital discharge. Geriatr Nurs. 2010; 31(3): 178-187. DOI: $10.1016 /$ j.gerinurse.2010.03.005.

28. Holland DE, Hansen DC, Matt-hensrud NN, Severson MA, Wenninger CR. Continuity of care: a nursing needs assessment instrument. Geriatr Nurs. 1998; 19(6): 331-334.

29. Carvajal A, Centeno C, Watson R, Martínez M, Sanz A. ¿Cómo validar un instrumento de medida de la salud?. Anales Sist San Navarra. 2011; 34(1): 63-72. 